\title{
Kolik des Pferdes aus osteopathischer Sicht
}

Was hat die Kolik mit dem Sternum zu tun?

Katja Eser

\section{Zusammenfassung}

Eine akute Kolik ist ein Notfall, der dringend der tierärztlichen Versorgung bedarf. Prophylaktisch ist die Osteopathie jedoch in der Lage, Verspannungen und Läsionen aufzudecken und zu behandeln, die das Entstehen einer Kolik begünstigen. Die Darstellung der möglichen muskulären, osteopathischen und energetischen Ursachen für eine Kolikneigung verdeutlicht die Komplexität des Krankheitsgeschehens. Das Sternum als „Mitte des Pferdes“ und mobiler Ansatzpunkt des Diaphragmas spielt in diesem Zusammenhang eine wichtige Rolle.

Der Begriff „Kolik“ ist in der Sprache von Pferdebesitzern, Tierärzten und Tiertherapeuten ein fest verankerter Begriff, obwohl er genau genommen keine Erkrankung bezeichnet, sondern ein Symptom: Bauchschmerzen. Mit einer Kolik zeigt ein Pferd einen körperlichen Zustand an, der von leichtem Unwohlsein bis hin zu stärksten Schmerzen geprägt sein kann.

Je nach Charakter des Pferdes und zugrunde liegender Erkrankung kann sich die Kolik zeigen in Zuständen höchster Unruhe, Schwitzen, dauerndem Hinlegen und Aufstehen, Flehmen, Bauchanschauen, mit einem Fuß gegen den Bauch schlagen, gegen die Wand werfen, aber auch in Form von Teilnahmslosigkeit, Fressunlust, vielleicht nur ein angedeutetes Einziehen der Bauchwand mit leicht veränderter Atmung. Diese Form der Kolik ist insofern besonders gefährlich, weil das Pferd kaum auf sich aufmerksam macht und die Dramatik des Krankheitsgeschehens manchmal nicht rechtzeitig erkannt wird.

Als mögliche Ursachen für eine Kolik kommen infrage [1]:

- Magen-Darm-Erkrankungen

- Leber- und Gallengangserkrankungen
- Erkrankungen der Harn- und Geschlechtsorgane

- Erkrankungen im Brust- und Schlundbereich

- Erkrankungen des Bewegungsapparats (Lumbago, Hufrehe)

- Hauterkrankungen

- Infektionskrankheiten (Tetanus, Tollwut, Bornavirose, Salmonellose, Milzbrand)

- parasitäre Erkrankungen

- Thromben/Embolien in Eingeweidegefäßen

- Wasser- und Futtermangel

Im akuten Fall ist die Kolik ein potenziell lebensbedrohlicher Zustand, der unbedingt einer tiermedizinischen Untersuchung und Behandlung bedarf!

Die dem Zustand der Kolik zugrunde liegenden möglichen Erkrankungen sind so vielfältig und zum Teil so drastisch, dass eine gründliche Ursachenforschung in Form von klinischer Untersuchung, Labor, evtl. Röntgen o. Ä. notwendig ist. Je nach Fall ist für Untersuchung und Behandlung gar ein Aufenthalt in einer Pferdeklinik erforderlich. Insofern hat der Pferdeosteopath und Tierheilpraktiker seine diagnostischen und therapeutischen Grenzen absolut realistisch einzuschätzen, um die Gesundheit und das Leben des Pferdes nicht zu gefährden! Andererseits können osteopathische, physiotherapeutische und naturheilkundliche Maßnahmen sehr wohl helfen, begleitend zur tiermedizinischen Versorgung die Genesung des Pferdes zu unterstützen, die Rekonvaleszenz zu fördern und Rezidive zu vermeiden.

\section{Lebenswirklichkeit des Pferdes}

Die der Kolik zugrunde liegenden möglichen Erkrankungen spielen sich auf der Grundlage der hohen Sensibilität und Labilität des Vegetativums des Pferdes ab. Dies kann als Folge nach sich ziehen, dass bereits Wetterumschwünge, Änderungen in der Fütterung, der Haltung oder der Stallroutine, Besitzer- oder Stallwechsel zum Auftreten von Koliksymptomen führen können.

Die Aufdeckung und Beseitigung eines etwaigen chronischen bzw. rezidivierenden Sympathikotonus, z.B. aufgrund schlechter Haltungsbedingungen, ungünstiger Herdenzusammensetzung mit ständigen Rangordnungskämpfen, schlechtem Umgang mit dem Pferd, physisch oder psychisch überfordernden Trainings, chronischer Schmerzzustände infolge mangelhafter Hufstellung/-bearbeitung oder eines schlecht sitzenden Sattels sind in diesem Zusammenhang wichtig für die Genesung und Prophylaxe.

In Herden- und Offenstallhaltungen ist besonderes Augenmerk darauf zu richten, dass auch die rangniedrigen Pferde ausreichend Zeit und Raum haben, in Ruhe ihr Raufutter in angemessenen Mengen und Zeitintervallen zu fressen und ebenso entspannt die Tränke aufzusuchen. Ebenso sind in Boxenhaltungen dem Pferd Sozialkontakte und ausreichend frei wählbare Bewegungsmöglichkeiten (Koppel, Paddock) anzubieten für ein gesundes vegetatives Gleichgewicht.

\section{Kolik aus Sicht der Osteopathie}

Aus osteopathischer Sicht ist interessant, dass es Pferde zu geben scheint, die eine sogenannte „Kolikneigung“ haben, d.h. sie sind deutlich gefährdeter, eine Kolik zu 
Tab. 1 Muskuläre Strukturen, die im Zusammenhang mit einer Kolikneigung relevant sind.

\begin{tabular}{|c|c|c|c|c|}
\hline & Ursprung & Ansatz & Innervation & Funktionen \\
\hline Diaphragma & $\begin{array}{l}\text { Pars lumbalis: L1-L4 } \\
\text { - Pars costalis: } \\
\text { Rippen 8-18 } \\
\text { - Pars sternalis: } \\
\text { Proc. xyphoideus }\end{array}$ & Centrum tendineum & $\begin{array}{l}\text { N. phrenicus aus nC5-nC7 } \\
\text { (Plexus brachialis) }\end{array}$ & $\begin{array}{l}\text { Hauptatemmuskel } \\
\text { Dassage der Bauchorgane } \\
\text { Aorta, Vena cava caudalis, } \\
\text { Ductus thoracis }\end{array}$ \\
\hline M. psoas minor & Th16-L4 & Os ilium & $\begin{array}{l}\text { Rr. ventrales der Segmente } \\
\text { Plexus lumbalis (nL2-nL6) }\end{array}$ & $\begin{array}{l}\text { Punctum fixum Hinterbein: } \\
\text { Extension von Becken und } \\
\text { LWS } \\
\text { - Punctum mobile Hinter- } \\
\text { bein: Flexion Becken und } \\
\text { Hüftgelenk } \\
\text { Besonderheit: Verlauf } \\
\text { direkt parallel zur Pars lum- } \\
\text { balis des Diaphragmas }\end{array}$ \\
\hline \multicolumn{5}{|l|}{ Mm. abdomines: } \\
\hline $\begin{array}{l}\text { M. transversus abdomi- } \\
\text { nis }\end{array}$ & $\begin{array}{l}\text { Rippenknorpel 7-18, } \\
\text { Procc. transversi L1-L6 }\end{array}$ & Linea alba & $\begin{array}{l}\text { segmental aus nTh4- } \\
\text { nTh18 } \\
\text { N. iliohypogastricus aus } \\
\text { nL1 } \\
\text { N. ilioinguinalis aus nL2/ } \\
\text { 3 (Plexus lumbalis) } \\
\text { N. genitofemoralis aus } \\
\text { nL2-nL4 (Plexus lumbalis) }\end{array}$ & $\begin{array}{l}\text { Exspirationshilfsmuskeln } \\
\text { - Bauchpresse bei Miktion, } \\
\text { Defäkation, Geburt } \\
\text { n Aufwölben der Wirbelsäule } \\
\text { n kontraktionsfähiger Trage- } \\
\text { gurt mit Anpassungsfähig- } \\
\text { keit an Volumen und Ge- } \\
\text { wicht der Bauchorgane } \\
\text { - Lateroflexion mit Rotation } \\
\text { des Rumpfes }\end{array}$ \\
\hline - M. rectus abdominis & $\begin{array}{l}\text { Sternum, Rippenknorpel } \\
\text { 4-9 }\end{array}$ & $\begin{array}{l}\text { Tendo praepubicus, Lig. } \\
\text { accessorium ossis femoris }\end{array}$ & & \\
\hline $\begin{array}{l}\text { M. obliquus externus } \\
\text { abdominis }\end{array}$ & $\begin{array}{l}\text { Außenfläche der Rippen } \\
6-18 \text {, Fascia thoracolum- } \\
\text { balis, Tuber coxae }\end{array}$ & $\begin{array}{l}\text { Linea alba, Lig. inguinale, } \\
\text { Tendo praepubicus (Ver- } \\
\text { lauf: kaudoventral) }\end{array}$ & & \\
\hline $\begin{array}{l}\text { M. obliquus internus } \\
\text { abdominis }\end{array}$ & Tuber coxae, Lig. inguinale & $\begin{array}{l}\text { Linea alba, Rippenknorpel 12- } \\
18 \text { (Verlauf: kranioventral) }\end{array}$ & & \\
\hline Mm. intercostales & $\begin{array}{l}\text { jeweils von Rippe zu Rippe } \\
\text { Verlauf: } \\
\text { Mm. intercostales ext.: kauc } \\
\text { Mm. intercostales int.: kran }\end{array}$ & entral & $\begin{array}{l}\text { segmental aus nTh1- } \\
\text { nTh18 }\end{array}$ & $\begin{array}{l}\text { Inspirationshilfsmuskeln } \\
\text { (Mm. intercostales externi) } \\
\text { Exspirationshilfsmuskeln } \\
\text { (Mm. intercostales interni) }\end{array}$ \\
\hline $\begin{array}{l}\text { M. erector spinae, Rumpf- } \\
\text { anteil: } \\
\text { M. semispinalis capitis } \\
\text { M. spinalis thoracis } \\
\text { M. longissimus thoracis } \\
\text { M. iliocostalis thoracis }\end{array}$ & $\begin{array}{l}\text { jeweils zwischen Procc. } \\
\text { transversi und Procc. spi- } \\
\text { nales über einzelne oder } \\
\text { mehrere Segmente, über } \\
\text { gerade oder schräge Ver- } \\
\text { läufe }\end{array}$ & & segmental aus nC3-nTh18 & $\begin{array}{l}\text { in Zusammenhang mit Kolik: } \\
\text { spannungsangepasstes } \\
\text { Punctum fixum für den-für } \\
\text { die Atemexkursion-mobilen } \\
\text { Thorax }\end{array}$ \\
\hline $\begin{array}{l}\text { Beckenboden: } \\
\text { - Diaphragma pelvis } \\
\text { - Diaphragma anale } \\
\text { - Diaphragma urogeni- } \\
\text { tale }\end{array}$ & $\begin{array}{l}\text { Lig. sacrotuberale latum, } \\
\text { proximale Vertebrae coc- } \\
\text { cygeae }\end{array}$ & $\begin{array}{l}\text { Vertebrae coccygeae, } \\
\text { Fascia coccygis, Anus, } \\
\text { Rectum, Vagina, Penis }\end{array}$ & $\begin{array}{l}\text { Rr. ventrales sacralis, } \\
\text { Nn. rectales kaudales aus } \\
\text { nS4/nS5, Nn perineales } \\
\text { proff. aus N. pudendus } \\
\text { (nS2-nS4) }\end{array}$ & $\begin{array}{l}\text { Miktion, Defäkation, Öffnen } \\
\text { und Schließen von Anus, } \\
\text { Urethra und Vagina, Erektion, } \\
\text { Schweifbewegungen, Beglei- } \\
\text { tung der Atembewegungen } \\
\text { des Diaphragma }\end{array}$ \\
\hline
\end{tabular}

bekommen, als andere Pferde. Was können die Ursachen sein, die dazu führen, dass ein Pferd immer wieder kolikartige Symptome zeigt? Um diese Frage zu beantworten, ist es sinnvoll, sich zu vergegenwärtigen, welche Strukturen ursächlich oder symptomatisch an den krampfartigen Bauchbeschwerden beteiligt sind und wie der „Bauch“ des gesunden Pferdes arbeitet.
Der Rumpf des Pferdes ist aufgeteilt in den Brust- und den Bauchraum. Im Brustraum befinden sich die Lungen und das Herz, im Bauchraum befinden sich Magen, Milz, Leber, Bauchspeicheldrüse, Dünndarm, Dickdarm mit seinen ventralen und dorsalen Lagen, seinen Flexuren am Diaphragma und am Becken. Richtung kaudal dicht ventral der Lendenwirbelsäule (LWS) finden sich die Nieren, im kleinen
Becken Eierstöcke, Eileiter, Gebärmutter, Harnblase.

Getrennt werden Brust- und Bauchraum voneinander durch das Diaphragma, das sich kuppelartig in den Brustraum (kranial konvex) wölbt. Diaphragma, Thorax mit der BWS, den Rippen und dem Sternum spielen eine nicht zu unterschätzende Rolle im Rahmen der Verdauung:

- Bei der Einatmung zieht sich das Diaphragma aktiv zusammen und schiebt 
dadurch alle Bauchorgane etwas nach kaudal. Der Beckenboden wölbt sich etwas nach kaudal und lässt die Kaudalverschiebung der Bauchorgane zu.

- Bei der Ausatmung (in Ruhe) entspannt sich das Diaphragma, die Bauchorgane und der Beckenboden heben sich wieder nach kranial.

Das Heben und Senken des Diaphragmas mit den entsprechenden biomechanischen Bewegungen der beteiligten knöchernen, muskulären und faszialen Strukturen unterstützt somit durch den erzielten Massageeffekt die natürliche Peristaltik der Verdauungsorgane. Alle Bauchorgane werden ständig entsprechend der Atembewegungen sanft gegeneinander verschoben, sodass ein Verkleben von Faszien, Gekröseanteilen, Darmschlingen o. Ä. vermieden wird.

\section{Mögliche muskuläre Ursachen}

Auf muskulärer Ebene finden sich die in ๑) Tab. 1 aufgeführten Strukturen mit der entsprechenden Innervation und den zugehörigen Wirbelsegmenten, die im $\mathrm{Zu}$ sammenhang mit einer Kolikneigung relevant sind.

\section{Muskelverspannungen}

Primäre Muskelverspannungen treten sehr häufig bei unphysiologischer Gymnastizierung des Pferdes, schlecht sitzendem Sattel, mangelhafter Hufstellung, einem unausbalancierten Reiter auf. Sekundär bzw. kompensatorisch treten Muskelverspannungen bei Läsionen der Wirbelsegmente, Extremitäten- oder Kiefergelenke, außerdem bei Störungen im kraniosakralen Bereich, insbesondere der Synchondrosis sphenobasilaris (SSB) auf.

Die Muskelverspannungen führen $\mathrm{zu}$ einer verringerten Atemexkursion entweder bzgl. des gesamten Thorax oder segmental. Die eingeschränkte Atemexkursion zieht eine reduzierte Darmmassage nach sich, was eine Obstipation begünstigen bzw. unterhalten kann. Dem Beckenboden ist hierbei große Aufmerksamkeit zu schenken.

\section{Obstipation}

Eine Obstipation kann Ursache für eine akute Kolik oder ein dauerndes Unwohlsein im Bauchraum sein. Dieses kann sich zeigen als

Tab. 2 Biomechanik der knöchernen thoraxbezogenen Strukturen, die im Zusammenhang mit einer Kolikneigung relevant sind.

\begin{tabular}{|c|c|c|}
\hline Struktur & Biomechanik der Einatmung & Biomechanik der Ausatmung \\
\hline Sternum & senkt sich nach ventrokranial & hebt es sich nach dorsokaudal \\
\hline $\begin{array}{l}\text { Rippen (insbesondere die } \\
\text { Atmungsrippen) }\end{array}$ & öffnen sich nach kraniolateral & $\begin{array}{l}\text { schließen sie sich nach kaudo- } \\
\text { medial }\end{array}$ \\
\hline $\begin{array}{l}\text { Th2-Th18 (insbesondere } \\
\text { Th9-Th18) }\end{array}$ & $\begin{array}{l}\text { Wirbelsegmente machen eine } \\
\text { Flexion }\end{array}$ & $\begin{array}{l}\text { Wirbelsegmente machen eine } \\
\text { Extension }\end{array}$ \\
\hline $\begin{array}{l}\text { Diaphragma mit seinem Be- } \\
\text { zug vom Proc. xyphoideus } \\
\text { sterni zur kranialen LWS }\end{array}$ & $\begin{array}{l}\text { Kontraktion und Sinken nach } \\
\text { kaudal }\end{array}$ & $\begin{array}{l}\text { Entspannung und Heben nach } \\
\text { kranial }\end{array}$ \\
\hline
\end{tabular}

- aufgezogene Bauchdecke

- Klemmigkeit im Rücken und der Hinterhand

- Unrittigkeit

- Verhaltensauffälligkeiten wie Sattelund Gurtzwang, Schnappen/Schweifschlagen beim Putzen des Bauches

- flache, oberflächliche Atmung

- schnellere Ermüdung und evtl. vermehrtes Schwitzen, da aufgrund eingeschränkter Atemexkursion die Sauerstoffversorgung des Blutes herabgesetzt ist

\section{Mögliche osteopathische Ursachen}

Auf knöcherner Ebene finden sich die in ๑ Tab. 2 dargestellten thoraxbezogenen Strukturen, die im Zusammenhang mit einer Kolikneigung relevant sind.

Bei Pferden mit sehr breitem und rundem Brustkorb kann die Biomechanik jeweils genau gegengleich stattfinden.

Funktionelle Läsionen in diesen Strukturen führen zu einer mechanischen Behinderung der Darmmassage. Kompensatorisch zieht dies Hypertonus oder Kontrakturen in den oben angesprochenen Muskeln, evtl. auch Hypotonus der entsprechenden Gegenseite nach sich, mit den oben bereits dargestellten Folgen.

\section{Foramen jugulare/Os temporale/} Atlas-Okziput/Kiefergelenk/Hyoid

Diese knöchernen Strukturen mit ihren entsprechenden muskulären Verbindungen, insbesondere $\mathrm{Mm}$. masseter und digastricus, parotidoauricularis, sternomandibularis, Ansatz des Lig. nuchae an der Protuberantia occipitalis externa sind im Rahmen der Untersuchung eines Pferdes mit Kolikneigung insofern von Bedeutung, als sie eine große topografische und funktionelle Nähe zum vegetativen Nervensystem haben. Insbesondere der N. vagus, der $\mathrm{X}$. Hirnnerv, mit seinen langen Ganglien zu den Verdauungsdrüsen und -organen, spielt im Rahmen des parasympathischen Systems eine große Rolle. Aufgrund seiner Anlage im Verlauf des Rückenmarks wird der parasympathische Anteil des vegetativen Nervensystems auch als das „kraniosakrale System“ bezeichnet. Dies ist zwar rein funktionell nicht identisch mit dem kraniosakralen System der Osteopathie, ist aber ein stimmiger Hinweis auf die Wirkungen im Körper in Form von Regeneration, Erholung, Auffüllen der körperlichen und energetischen Reserven, Selbstheilung, und Selbstregulation.

Störungen in diesen Strukturen können zu Dysbalancen im vegetativen Nervensystem führen mit der Folge eines chronisch oder rezidivierend auftretenden Sympathikotonus. Besonders die Aktivität des Diaphragmas und damit auch des Sternums reagieren ausgesprochen sensibel auf ein Missverhältnis zwischen Sympathikus und Parasympathikus.

\section{Synchondrosis sphenobasilaris}

Die Schädelbasis, die sich aus der Gelenkfläche zwischen dem Corpus occipitalis und dem Corpus ossis basisphenoidalis zusammensetzt, ist der knöcherne „Motor" des kraniosakralen Systems der Osteopathie. Die gesunde Funktion der SSB ist ausschlaggebend für die freie Fluktuation des Liquors in Gehirn und Rückenmark und die physiologische Ausbreitung des kraniosakralen Rhythmus im ganzen Körper.

Durch die unmittelbare Nähe der Sella turcica („Türkensattel“) oberhalb des Sphenoids kommt der Synchondrosis spheno- 
basilaris (SSB) außerdem eine wichtige Rolle im Zusammenhang mit der Hypophyse zu, also der physiologischen Regulation zwischen Hormon- und Nervensystem.

Neben der Begründung einer Kolikneigung können sich Dysbalancen der SSB bei drastischen Befunden bzw. bei lang anhaltenden Läsionen zeigen als

- sichtbare Asymmetrien im Gesicht des Pferdes, z. B. verschieden groß erscheinende Augen, unterschiedlich stehende Ohren, asymmetrische Maulwinkel und Nüstern,

- sichtbare Asymmetrien der Extremitätenstellungen, z.B. beide Beine einer Körperseite in Außenrotation, die gegenüberliegenden Beine in Innenrotation und/oder

• Verhaltensauffälligkeiten in Form von unerklärlichen Stimmungsschwankungen (z.B. plötzliches aggressives Verhalten gegenüber Besitzer oder Herdenmitglied), Apathie, Unruhe, Konzentrationsstörungen im Training, Koordinationsstörungen („Mein Pferd haut sich oft den Kopf an.“, „Mein Pferd bleibt oft mit der Hüfte irgendwo hängen.“)

\section{Faszien}

Die Faszien haben eine herausragende Bedeutung für die Gesamtkörperfunktion und -integration. Sie umhüllen alle einzelnen Körperstrukturen, größere Muskelgruppen, funktionelle Gewebseinheiten.

Sie dienen der

- Abgrenzung der einzelnen Strukturen voneinander,

- Weitergabe von Spannungen/Lösungen von einer Struktur zur anderen,

- ernährungsmäßigen und fluiden Versorgung und Drainage dieser Strukturen und

- der reibungslosen und harmonischen Integration der verschiedenen Körpersysteme untereinander.

Jeder Zug auf eine Faszie verändert deren Struktur und damit auch deren Elastizitätsverhalten, ähnlich wie bei einem Gummiband, das nach dem ersten fehlerhaft ausgeübten Dehnzug nicht mehr 100-prozentig seine ursprüngliche Form und Kürze einnimmt. Auf diese Weise finden sich alle (Fehl-)Spannungen, denen der Körper jemals ausgesetzt war, in den Faszien ein Leben lang wieder (= Gedächtnis der Faszie).

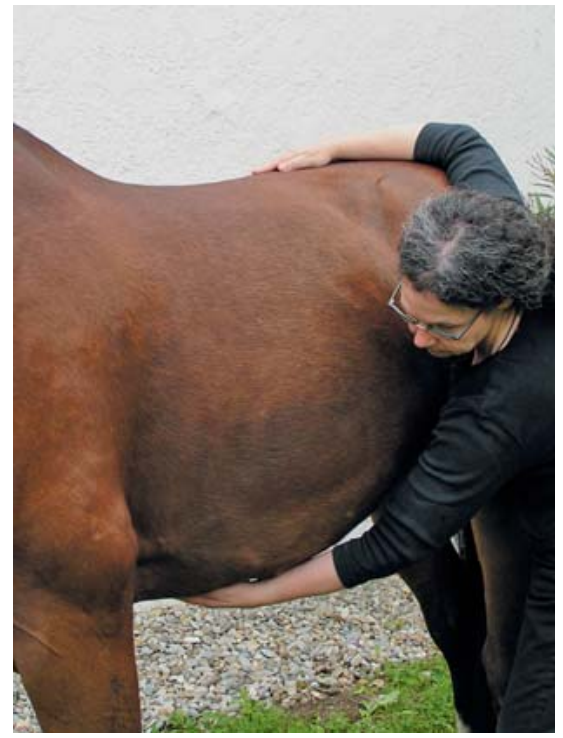

Abb. 1 Release des Diaphragmas zwischen Proc. xyphoideus sterni und LWS. (C) Eser K. Checkliste Osteopathie Pferd. Stuttgart: Sonntag in MVS Medizinverlage; 2011.

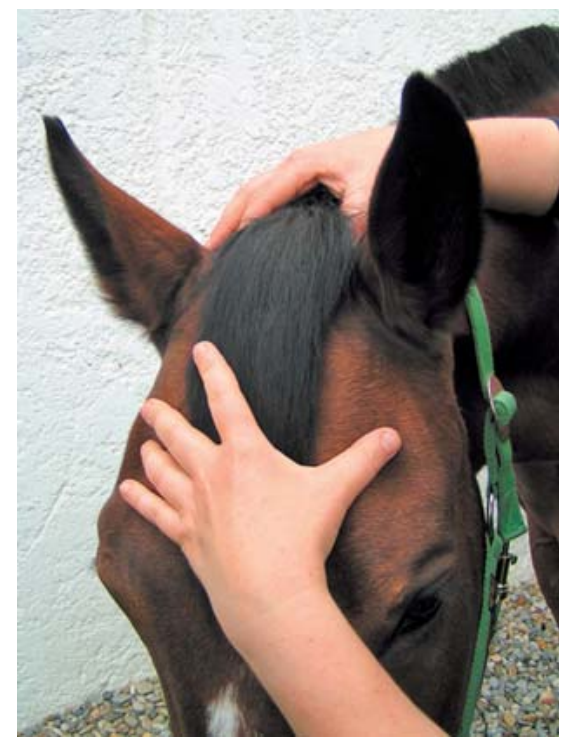

Abb. 2 Lösung von Läsionen der SSB. (C) Eser K. Checkliste Osteopathie Pferd. Stuttgart: Sonntag in MVS Medizinverlage; 2011.

Tab. 3 Meridiane, die im Zusammenhang mit einer Kolik stehen.

\begin{tabular}{|c|c|c|c|}
\hline Meridian (Zuordnung) & zuständig u.a. für & Alarmpunkt & Referenzwirbel \\
\hline Magen (Yang) & $\begin{array}{l}\text { Nahrungsaufnahme und } \\
\text { Verdauung }\end{array}$ & KG 12 & $\mathrm{C} 1-\mathrm{C} 8$ \\
\hline Dünndarm (Yang) & $\begin{array}{l}\text { Verdauung, Absorption von } \\
\text { Wasser und Nährstoffen }\end{array}$ & KG 4 & Th5-Th12, L2-L4 \\
\hline Dickdarm (Yang) & $\begin{array}{l}\text { Verdauung, Absorption von } \\
\text { Flüssigkeit }\end{array}$ & Ma 25 & L4-S3 \\
\hline Lunge (Yin) & $\begin{array}{l}\text { rhythmische Körperfunk- } \\
\text { tionen (Atmung, Peristaltik) }\end{array}$ & Lu 1 & $\begin{array}{l}\text { C5-C7 (Nervenwur- } \\
\text { zeln für N. phrenicus } \\
\rightarrow \text { Diaphragma) }\end{array}$ \\
\hline $\begin{array}{l}\text { 3-fach Erwärmer } \\
\text { (Yang) }\end{array}$ & $\begin{array}{l}\text { „Betriebstemperatur“ der } \\
3 \text { Räume Lunge, Verdau- } \\
\text { ungsorgane, Genitale }\end{array}$ & KG 5 & C4-C6 \\
\hline
\end{tabular}

$\mathrm{C}=$ Halswirbel, $\mathrm{KG}=$ Konzeptionsgefäß, Ma = Magenmeridian, $\mathrm{L}=$ Lendenwirbel, $\mathrm{Lu}=$ Lungenmeridian, Th = Brustwirbel,

\section{Mögliche energetische Ursachen}

Die Sichtweise der Traditionellen Chinesischen Medizin (TCM) bietet einen weiteren Aspekt der Annäherung an die möglichen Zusammenhänge ständiger oder wiederkehrender „Bauchschmerzen“. Eine Fülle oder Leere in den in \$ Tab. 3 aufgeführten Meridianen kann in diesem Fall ursächlich bzw. symptomatisch sein.

\section{Osteopathische Behandlungs- möglichkeiten}

Nach Erfassung der individuellen Befunde des betroffenen Pferdes kommen die entsprechenden Behandlungsgriffe und -techniken zur Anwendung [2]:
- Detonisation der hypertonen Muskulatur durch Massagegriffe, Stresspunktbehandlung, Dehnungen

- Lösung faszialer Verklebungen durch Ausziehen der oberflächlichen Faszien und Myofascial Release

- Lösung der Blockierungen des Sternums, der Rippen, der BWS durch direkte und indirekte Techniken

- Öffnung des Foramen jugulare durch Ear-Pull

- Atlas-Okziput-Release

- Release des Diaphragmas zwischen Proc. xyphoideus sterni und Lendenwirbelsäule (@ Abb. 1)

- Lösung von Läsionen der SSB (๑ Abb. 2) 
Die Wiederherstellung der muskulären Balance der angesprochenen Muskelketten, die Lösung der beteiligten knöchernen Strukturen und Faszien sowie die Harmonisierung des vegetativen Nervensystems über die SSB stellen für ein anfälliges Pferd ein vielversprechendes Behandlungskonzept dar, um das Auftreten von Koliken zu vermindern bzw. deren klinische Verläufe abzumildern.

\section{Summary}

\section{Osteopathic view of colic in horses: How is colic linked to sternum?}

Acute colic is an emergency case that requires veterinarian care. Prophylactically, osteopathy is capable of detecting tensions and lesions that lead to the outbreak of colic. The presentation of the possible muscular, osteopathic and energetistic causes of colic tendency illustrates the complexity of the occurrence of the disease. The sternum as the "middle of the horse" and mobile point of the diaphragm, plays an important role with regard to the disease.

Dey words

osteopathy - horse - colic

() Literatur

[1] Dietz O, Huskamp B. Handbuch Pferdepraxis. Stuttgart: Enke in MVS Medizinverlage; 2006

[2] Eser K. Checkliste Osteopathie Pferd. Stuttgart: Sonntag in MVS Medizinverlage; 2011

Online zu finden unter

http://dx.doi.org/10.1055/s-0032-1328411

Katja M.-L. Eser

Human-Physiotherapeutin, Pferde-Osteopathin Judmannstr. 1 85049 Ingolstadt

E-Mail: Katja.Eser@horsehealing.de

Jahrgang 1967, 1994 Amtsarztprüfung Humanheilpraktikerin, 2002 Staatsexamen Humanphysiotherapie, 2004 Tierheilpraktikerin (ATN), 2006 Pferdeosteopathie (EPOS), 2009 Equine Lymphdrainage nach Prof. Rautenfeld (TiHo Hann.). Seit 1994 in selbstständiger Naturheilpraxis, seit 2002 zusätzlich freiberuflich in Physiotherapiepraxis tätig. Seit 2006 selbständige Fahrpraxis mit Schwerpunkt Osteopathie und Gymnastizierung für Pferd und Reiter. 2007-2010 Dozentin für Pferdetherapeuten in Ingolstadt, seit 2012 in Geretsried und Worpswede. 2011 Veröffentlichung des Buches „Checkliste Osteopathie Pferd“ im Sonntag Verlag 